\title{
The Tomographic atom probe: salient results and recent breakthroughs
}

\author{
A.Menand*, B. Deconihout*, P. Pareige*, F. Vurpillot* and D. Blavette*, \\ *Groupe de Physique des Matériaux - UMR CNRS 6634, Institute for Materials Research, \\ UFR Sciences et Techniques, B.P. 12, 76801 Saint Etienne du Rouvray - Cedex, France
}

Because the screening distance of the applied electric field at the sample surface is less than an atomic interspacing in metallic sample, Atom Probe Field Ion Microscopy (APFIM) makes it possible atomic-layer-by-atomic-layer chemical analysis of materials [1]. In 1983 this ultimate resolution was used to performed the first determination of occupation site frequency in an ordered phase, the fundamental and superlattice layers of the $\gamma^{\prime}$-phase $\left(\mathrm{Ni}_{3} \mathrm{Al}\right.$-type $)$ were clearly resolved [2].

However, plane by plane analysis of ordered alloys using classical 1D atom probe is rather difficult to perform in a quantitative way. The evaporation of several identical atoms on the same electric pulse often occurs. This artifact so-called pile up effect leads to biased composition data as multiple events are detected as a unique event. The first attempt to cope with this problem was made by the Rouen team in 1985 using a four-sector detector. This first multiple anode device used in atom probe techniques allowed the detection of multiple events and was also the first device giving a spatial information from composition profiles supplied by each detection sector [3].

APFIM is basically an ion projection microscope. Panitz obtained the first images of the distribution of selected species with the imaging atom-probe [4]. However only the invention of Position Sensitive Atom Probe (PoSAP) in 1988 by the Oxford group allowed for the first time to simultaneously record the two pieces of information brought about by the evaporated ions i.e. their time of flight leading to their chemical characterization and their impact position onto the detector giving their position at the tip surface. The use of a 2D detector thus led to the first 3D analytical images at an atomic scale [5]. However the position sensitive detector used in PoSAP was not able to record simultaneous impact, which prevented quantitative analyses to be conducted.

Both experiences of the four-sector detector and the PoSAP were essential in the development at the University of Rouen of the Tomographic Atom-Probe (TAP) that gives 3D atomic-scale quantitative images [6]. Among the many results in materials science the most impressive are probably the first 3D image of a Cottrell atmosphere [7] shown in Fig. 1 or the neutron-induced clusters in pressure vessel steel [8] as shown in Fig.2.

Because fast electric pulses can not be properly transmitted to the specimen apex, the APFIM is limited to metallic materials. The invention of the pulsed-laser atom-probe by Tsong et al. in 1980 [9] was a first step to the analysis of poor conductive materials such as semiconductors. Unfortunately because the available pulsed-lasers at that time were in the nanosecond range the evaporation was thermally assisted. This led to difficult and non-quantitative analyses of alloys. We showed recently that the use of ultra-fast laser pulses makes possible to bring high electric fields to the specimen apex whatever its conductivity. The field evaporation assisted by the laser field is produced without any significant thermal effect [10]. Fig. 3 shows a 3D image of an AlMgSi alloy obtained by femto-second pulsed-laser TAP. In addition, the mass resolution is very high, see Fig 4, making possible to design a super straight high-resolution $3 \mathrm{D}$ atom probe. 


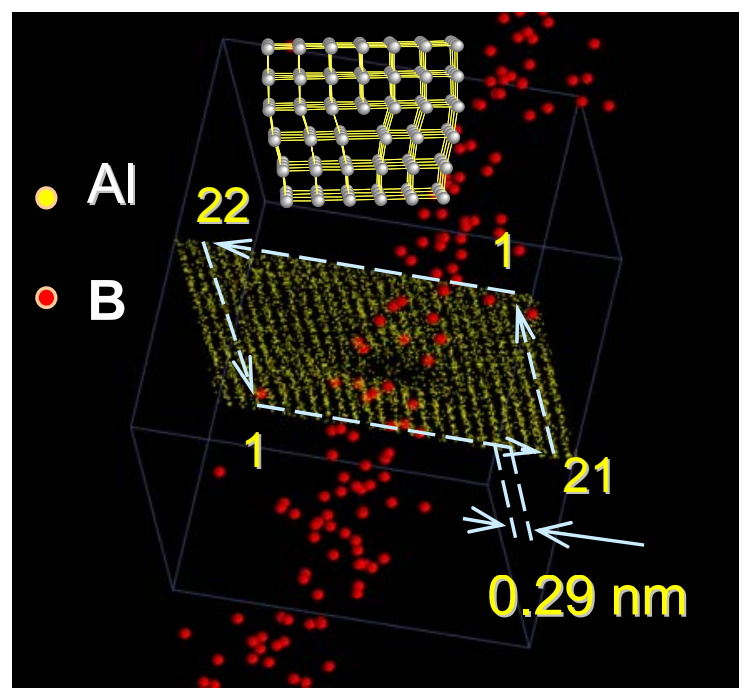

FIG. 1. 3D maps of aluminium and boron atoms show the presence of a boron enriched Cottrell atmosphere along an edge dislocation in a B2 ordered FeAl alloy.

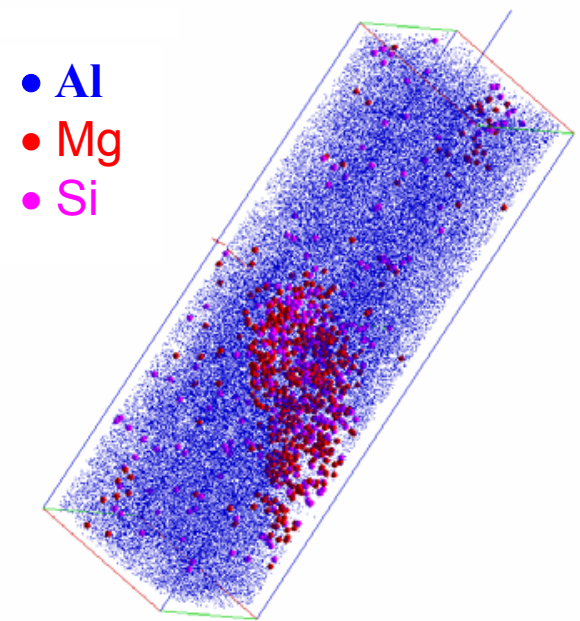

FIG. 3. Laser assisted 'IAP image of an AIMgSi alloy showing a well resolved needle-like $\beta$ "' precipitate.

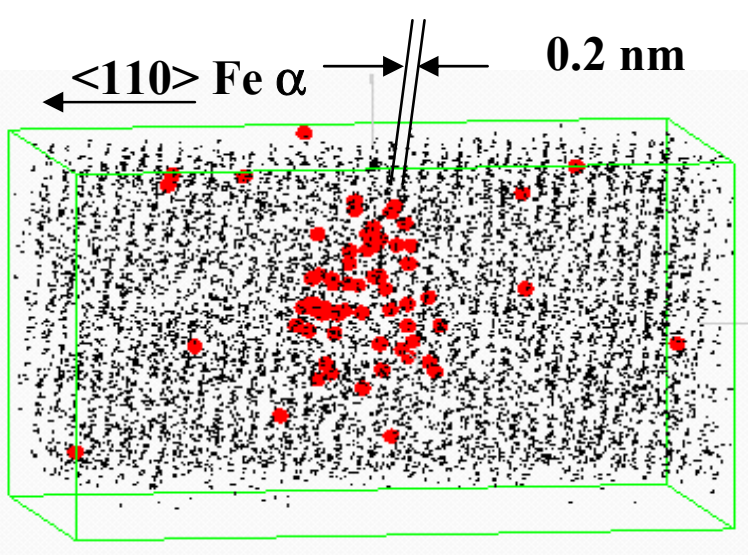

FIG. 2. Atomic observation of a copper rich cluster formed under neutron irradiation in pressure vessel steel of a nuclear reactor.

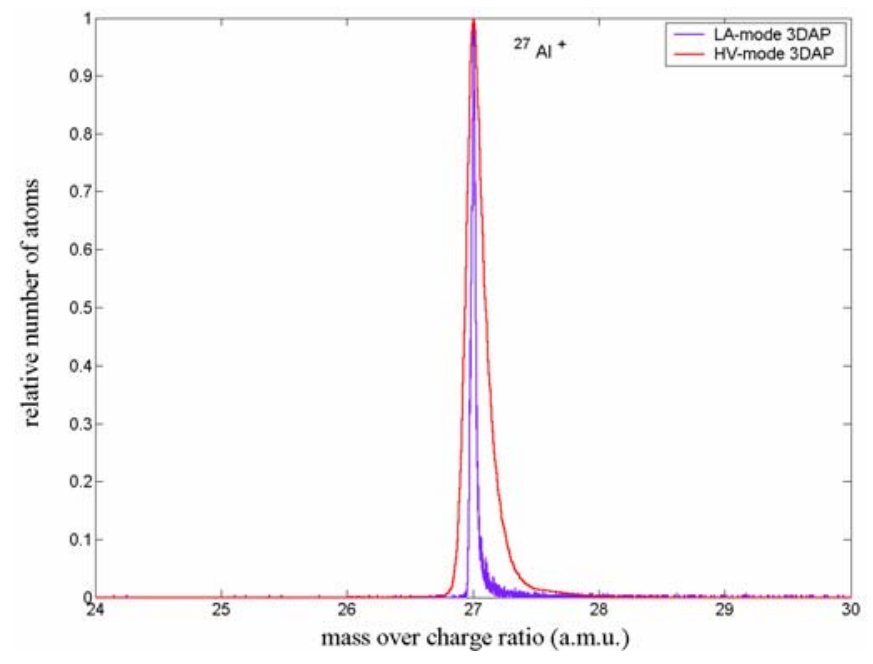

FIG. 4. Mass spectra of an AlMgSi alloy obtained in the laser assisted mode and in the HV pulsed mode.

\section{References}

[1] T.T. Tsong and E. W. Müller, J. Appl. Phys., 45, (1974) 2367

[2] D. Blavette and A. Bostel, Acta Metall, 32 (1984) 811-816.

[3] D. Blavette, A. Bostel and J.M. Sarrau, J. de Physique, 47 (Suppl. 3) (1886) C2-473.

[4] J.A. Panitz, Rev. Sci. Instrum., 44 (1973) 1034.

[5] A. Cerezo, T.J. Godfrey and G.D.W. Smith, Rev. Sci. Instrum., 59 (1988) 862-866.

[6] D. Blavette et al., Rev. of Scientific Instrum., 64 (1993) 2911-2919

[7] D. Blavette et al., Science, 286 (1999) 2317 - 2319.

[8] P. Pareige et al., Scripta Metall. et Mater., 33 (1995) 1033-1036

[9] P. Pareige et al., Phil. Mag;, 85 (2005) 429-441.

[10] G. L. Kellogg and T. T. Tsong, J. Appl. Phys., 51 (1980) 1184-1193.

[11] B. Deconihout et al., to appear in Surface and Interface Analysis. 